\title{
Consumer Prices Index and Retail Prices Index: the 2011 basket of goods and services
}

\author{
Philip Gooding \\ Office for National Statistics
}

\section{Summary}

The 'shopping baskets' of items used in compiling the Consumer Prices Index (CPI) and Retail Prices Index (RPI) are reviewed each year. Some items are taken out of the baskets and some are brought in to make sure the CPI and RPI are up to date and representative of consumer spending patterns. This article describes the review process, explains how and why the various items in the CPI and RPI baskets are chosen, and discusses the main changes to the contents of the CPI and RPI baskets for 2011 from the 2010 price collection. Similar articles have been published in previous years. This article also describes one other change relating to the way in which prices of seasonal items are measured in the CPI and RPI.

\section{The shopping basket}

A convenient way of thinking about both the CPI and RPI is to imagine a 'shopping basket' containing those goods and services on which people typically spend their money. As the prices of the various items in the basket change over time, so does the total cost of the basket. Movements in the $\mathrm{CPI}$ and RPI represent the changing cost of this representative shopping basket.

In principle, the basket should contain all consumer goods and services purchased by households, and the prices measured in every shop or outlet that supplies them. In practice, both the CPI and $\mathrm{RPI}$ are calculated by collecting a sample of prices for a selection of representative goods and services in a range of UK retail locations. Currently, around 180,000 separate price quotations are used every month in compiling the indices, covering over 650 representative consumer goods and services for which prices are collected in around 150 areas throughout the UK.

Within each year, the CPI and RPI represent the changing cost of a basket of goods and services of fixed composition, quantity and quality. In practice, this is achieved by (a) keeping constant the sample of representative goods and services; (b) applying a fixed set of weights to price changes 
for each of the items such that their influence on the overall index reflects their importance in the typical household budget; and (c) taking care to ensure that replacements for brands that are no longer stocked in an individual shop are of comparable quality. In this way, changes in the CPI and RPI from month to month reflect only changes in prices, and not ongoing variations in consumer purchasing patterns.

However, the contents of the CPI and RPI baskets of goods and services and their associated expenditure weights are updated annually. This is important in helping to avoid potential biases that might otherwise develop over time - for example, due to the development of entirely new goods and services, or the tendency for consumers to move away from buying goods and services which have risen relatively rapidly in price. For example, if the price of tea rose dramatically during one year, consumers might switch their spending towards coffee, making it necessary to adjust the expenditure weights accordingly in the following year.

These procedures also help to ensure that the indices reflect longer-term trends in consumer spending patterns. For example, the proportion of household expenditure devoted to household services has broadly risen overall over the last 25 years. This is reflected both in an increasing weight for this component in the $\mathrm{CPI}$ and RPI, and the addition of new items in the basket to improve measurement of price changes in this area: examples include internet subscriptions, playgroup and nanny fees.

For the RPI, changes to the items and weights are introduced in the February index each year, but prices are collected for both old and new items in January. This means that the figures for each year can be 'chain linked' together to form a long-run price index spanning many years. In other words, price changes into January are based on the old basket and weights, and price changes between January and February, and beyond, are based on the new basket and weights. This procedure ensures that the annual changes to the basket and weights have no impact on estimated changes in prices as measured by the indices. The same basic approach is adopted in the $\mathrm{CPI}$ although, for technical reasons, it is necessary to chain link the published index twice each year rather than only once as in the RPI ${ }^{1}$.

ONS (2010a) 'Consumer Price Indices - a Brief Guide' provides a helpful introduction to the concepts and procedures underpinning the compilation of the CPI and RPI indices. These are described in much greater detail in ONS (2010b) 'Consumer Price Indices - Technical Manual'.

\section{Representative items}

There are some individual goods and services where typical household spending is so large that they merit inclusion in the basket in their own right: examples include petrol, and electricity and gas supply. However, it would be both impractical and unnecessary to measure price changes of every item bought by every household in compiling the CPI and RPI.

More commonly, a sample of specific goods and services has to be selected that gives a reliable measure of price movements for a broader range of similar items. For example, price changes for 
garden spades might be considered representative of price changes for other garden tools. The selection of these representative items is judgmental since the significant difficulties involved in defining an adequate sampling frame (that is, a list of all the individual goods and services bought by households) restrict the use of traditional random sampling methods when choosing the representative items.

For each product grouping, a number of items are selected whose price movements, when taken together, provide a good estimate of the overall change in prices for the group. For example, there are around 20 representative items in the CPI 'furniture and furnishings' class, from bedroom wardrobes to kitchen units, whose prices are used to calculate an overall estimate of price changes for all furniture products.

The prices collected for each product group are then combined to produce the overall CPI and RPI, with weights proportional to total expenditure on the entire product group. So the weight given to 'furniture and furnishings' in the CPI shopping basket, or 'furniture' in the RPI basket, reflects average household spending on all furniture products as opposed to spending on the basket items only. Similarly, the weight of garden spades would be derived from all spending on garden tools.

\section{Table $1 \quad$ High level RPI weights since 1987}

Weights are specified as parts per 1000 of the all items RPI

\begin{tabular}{lccccccccc}
\hline RPI GROUP & $\mathbf{1 9 8 7}$ & $\mathbf{1 9 9 2}$ & $\mathbf{1 9 9 7}$ & $\mathbf{2 0 0 2}$ & $\mathbf{2 0 0 7}$ & $\mathbf{2 0 0 8}$ & $\mathbf{2 0 0 9}$ & $\mathbf{2 0 1 0}$ & $\mathbf{2 0 1 1}$ \\
\hline Food & 167 & 152 & 136 & 114 & 105 & 111 & 118 & 112 & 118 \\
Catering & 46 & 47 & 49 & 52 & 47 & 47 & 50 & 47 & 47 \\
Alcohol & 76 & 80 & 80 & 68 & 66 & 59 & 63 & 64 & 60 \\
Tobacco & 38 & 36 & 34 & 31 & 29 & 27 & 27 & 27 & 28 \\
Housing & 157 & 172 & 186 & 199 & 238 & 254 & 236 & 237 & 238 \\
Fuel and light & 61 & 47 & 41 & 31 & 39 & 33 & 49 & 40 & 42 \\
Household goods & 73 & 77 & 72 & 73 & 66 & 66 & 70 & 67 & 65 \\
Household services & 44 & 48 & 52 & 60 & 65 & 64 & 61 & 59 & 63 \\
Clothing and footwear & 74 & 59 & 56 & 51 & 44 & 42 & 39 & 40 & 44 \\
Personal goods and services & 38 & 40 & 40 & 43 & 39 & 41 & 41 & 41 & 38 \\
Motoring expenditure & 127 & 143 & 128 & 141 & 133 & 133 & 121 & 144 & 137 \\
Fares and other travel costs & 22 & 20 & 20 & 20 & 20 & 20 & 20 & 20 & 20 \\
Leisure goods & 47 & 47 & 47 & 48 & 41 & 38 & 38 & 37 & 36 \\
Leisure services & 30 & 32 & $59^{2}$ & 69 & 68 & 65 & 67 & 65 & 64 \\
\hline & & & & & & & 54
\end{tabular}

1. Depreciation costs were added to the housing group in 1995

2. Foreign holiday costs were added to the leisure services group in 1993, followed by UK holidays a year later. Source: Retail Prices Index 
These expenditure weights are also updated annually so that the indices reflect current spending patterns. In line with usual practice, CPI class weights were updated with effect from the January 2011 index, and RPI section weights will be revised with effect from the February index, at which point the weights for the more detailed (unpublished) item indices will also be revised. A brief comparison of high level RPI weights since 1987 is shown in Table 1, including the new weights for 2011. The table illustrates that over the period there are some clear shifts in expenditure. Broadly speaking, weights for services have increased while those for goods have decreased. A more detailed article on changes to the published CPI and RPI weights will be published on the National Statistics website in April 2011.

The contents of the CPI and RPI baskets are very similar, although the precise weights attached to the individual items in each index differ ${ }^{2}$. There are, however, some differences between the baskets. For example, the RPI basket includes a number of items chosen to represent owneroccupier housing costs, including mortgage interest payments and depreciation costs, all of which are excluded from the CPI. These differences are summarised in ONS (2010c) 'Differences between the RPI and CPI Measures of Inflation' and described in greater detail in Roe and Fenwick (2004), 'The New Inflation target: the Statistical Perspective'.

\section{Selecting the representative items}

A number of factors need to be taken into account when choosing representative items. Of course, the items must be easy to find by price collectors, so ensuring that estimates of price change are based on an adequate number of price quotes collected throughout the UK. Since the CPI and RPI are based on the cost of a fixed in-year basket of goods and services, ideally they should also be available for purchase throughout the year. However, availability of some food and clothing items is clearly seasonal, and so these goods require a slightly different treatment in the indices.

The number of items chosen to represent each product group within the CPI and RPI depends both on the weight (expenditure) of the group and also the variability of price changes between the various items that could be selected to represent the group (reflecting, for example, the diversity of products available). Intuitively, it makes sense to choose more items in product groups where spending is high; this helps to minimise sampling variability in the estimate of price change for high-weighted groups, and therefore in the overall price index. However, if price movements of all possible items in the group are very similar, it is sufficient to collect prices for only a few ${ }^{3}$. By contrast, if price movements of all the possible items are very different, prices will be needed for many representative items to get a reliable overall estimate of price change for the group.

Based on this, the allocation of items to broad commodity groups can be analysed, as shown for the 12 divisions of the CPI in Table 2, and the balance used as an anchor for the annual review of the basket. The significant allocation of items to the food division relative to its index weight, for example, is partly explained by the relatively high variation in observed price changes between the individual goods in this area. Conversely, a smaller proportion of items relative to index weight is allocated to the restaurants and hotels division, reflecting greater similarity in observed price changes. In some cases, such as transport and housing, apparent low allocations of items are explained by the presence of some dominant individual items (for example car purchase and motor 
fuels, and housing rents respectively). Here, the case for adding further items to improve coverage of these divisions' remaining index weights is much weaker - instead, it is far more important to ensure that the sampling of prices for these heavily weighted items is as comprehensive as possible.

\section{Table $2 \quad$ Allocation of items to CPI divisions in 2011}

\begin{tabular}{llccc}
\hline & & $\begin{array}{c}\text { CPI weight } \\
\text { (per cent) }\end{array}$ & $\begin{array}{c}\text { Observed } \\
\text { variation in price } \\
\text { changes }\end{array}$ & $\begin{array}{c}\text { Representative items }{ }^{2} \\
\text { (per cent of total) }\end{array}$ \\
\hline 1 & Food \& non-alcoholic beverages & 11.8 & High & 23 \\
2 & Alcohol \& tobacco & 4.2 & Low & 3 \\
3 & Clothing \& footwear & 6.2 & High & 5 \\
4 & Housing \& household services & 11.9 & High & 11 \\
5 & Furniture \& household goods & 6.1 & Medium & 3 \\
6 & Health & 2.4 & Low & 6 \\
7 & Transport & 15.9 & High & 1 \\
8 & Communication & 2.6 & High & 17 \\
9 & Recreation \& culture & 14.7 & High & 1 \\
10 & Education & 1.8 & High & 8 \\
11 & Restaurants \& hotels & 12.0 & Low & 11 \\
12 & Miscellaneous goods \& services & 9.4 & High & \\
\hline & & & & \\
\hline
\end{tabular}

1. Based on an analysis of variation in price changes between the individual items chosen to represent each division in the period 2004-2008.

2. These figures should be treated as providing only a broad indication of the allocation of items to the 12 $\mathrm{CPI}$ divisions. For example, the sample of prices underpinning an existing item might easily be stratified in some way to form two or more distinct items; conversely, items could be merged to form a single item representing a wider, more heterogeneous, spending category. A specific example of this is the item 'University tuition fees'. This is classified as one item but the index takes into account prices for several hundred courses, including undergraduate, postgraduate, part-time etc.

The analysis also helps to highlight those areas of the index which might benefit most from improved coverage, such as miscellaneous goods and services. The current allocation of items to the division is broadly comparable to its index weight but variation in price changes appears relatively high, possibly reflecting the diversity of goods and services covered by this division. As discussed later, this type of analysis has motivated some of the changes to the basket introduced in 2011. Conversely, it also helps to highlight areas where there is scope to remove items from the basket without any significant loss of precision in the indices. It is important that growth in the overall size of the basket is limited each year so that production costs and processing times are contained. 
Such analysis cannot tell us which items should be priced, and so choosing a particular set of items to represent each area remains a matter of judgement. CPI and RPI commodity groupings are regularly reviewed with the aim that all significant items or distinct markets where consumers' expenditure exceeds around $£ 400$ million annually are explicitly represented in the basket, except where those items are judged to be adequately represented by other items in the basket ${ }^{4}$. Conversely, where spending on items falls below the $£ 100$ million mark, there should be good reason for their continuing inclusion in the basket. For example, while spending on acoustic guitars and power drills is relatively low, both are included in the basket to represent wider markets (musical instruments and electrical tools respectively) that would otherwise not be covered explicitly. Trends in expenditure, as well as the latest available figures, help to inform the decisions in all cases.

This focus on expenditures in determining the contents of the basket partly reflects the data that are available describing household spending patterns. One major source of information comes from the diaries and questionnaires filled in by people taking part in the ONS Living Costs and Food Survey, a continuous survey of around 6,000 households each year. This is supplemented by detailed analyses of trends presented by market research companies, trade journals and in press reports. Changes in the retail environment are also reported to ONS by the price collectors, and together these various sources of information help to ensure that the goods and services that the average household spends its money on are appropriately represented in the CPI and RPI baskets.

It is very important to note that the contents of the basket and, in particular, changes from one year to the next should not be given significance beyond their purpose as representative items used in estimating retail price changes. Changes to the basket will reflect evolving consumer tastes, but only over a long run of years. In any particular year, changes to the basket will reflect a range of considerations such as practical experience in collecting prices, the desire to improve coverage in high spending areas, or analysis that suggests that estimated price changes could be improved at the margin by varying the number or type of representative items collected. Indeed, within each product grouping there is usually a point at which the exact number, choice of items and the precise weights attached to them becomes a matter of relatively fine judgement. At this detailed level, it is unlikely that such choices would have any significant impact on the CPI and RPI indices. For example, a selection of specific household appliances has been chosen to represent spending on small electrical goods, including irons and kettles. However, other representations would clearly be possible and equally valid.

It should also be noted that the vast majority of the 650 or so representative items remain unchanged in 2011.

In summary, selection of representative items is based on a number of factors, including:

- ease of finding and pricing the product

- availability throughout the year

- variability of prices within a class

- analysis of balance across the basket

- amount spent on a particular item or the group of items 


\section{Changes to the basket in 2011}

Changes to the basket of goods and services this year are being introduced with the February 2011 consumer price indices published on 22 March 2011; that is, monthly changes in prices from February 2011 to January 2012 inclusive are estimated with reference to the updated basket. The basket will be updated again at the same time next year.

New additions to the basket in 2011 and those items removed are set out in Tables $\mathbf{3}$ and $\mathbf{4}$, together with a brief summary of the motivation for these changes. As the tables make clear, these motivations are diverse. As in previous years, changes to the basket in 2011 certainly should not be viewed as a simple indicator of those products or services whose popularity has either grown or fallen significantly over the past year. Note that all of the changes to the basket this year affect both the CPI and RPI indices.

The bullet points below give a brief summary and explanation of the themes behind the changes to the basket for 2011 :

- A number of new items are introduced to represent specific markets where consumer spending is significant, and existing items in the basket may not adequately represent price changes for such goods. For example, craft kits are being introduced to represent a distinct sector not previously covered in the games, toys and hobbies class. Oven-ready joints are also included for the first time to reflect a longer-term move to prepared food and away from traditional joints of meat.

- Two high-technology goods are being introduced this year that can be seen as representing evolving trends: smart phone handsets and mobile phone applications are introduced to capture price changes in these rapidly expanding markets.

- In addition to introducing items to represent distinct sectors or markets, a number of items have been introduced to diversify the range of products collected for already established groupings, usually where spending is significant. For example, hardback fiction books widen coverage of the books sector. Similarly the number of television items in the basket has been increased from two to three, reflecting the greater diversity in size now available and, in particular, to better represent the upper end of the range where they form part of home entertainment systems.

- Analysis of the broad balance of the existing sample of representative items across the CPI highlighted a need to improve coverage of price changes for a number of CPI classes. These areas include:

- Appliances and products for personal care (12.1.2/3), with a new item covering hair conditioner to supplement existing toiletries such as toothpaste and deodorant;

- Other services not elsewhere covered (12.7.0), where dating agency fees have been added to broaden coverage in a particularly diverse grouping. 


\section{Table $3 \quad$ Additions to the basket in 2011}

\begin{tabular}{|c|c|c|c|}
\hline CPI Class & RPI Section & New Item & Notes \\
\hline 01.1.2 Meat & 2110 Other Meat & Oven-ready joint & $\begin{array}{l}\text { Replaces pork shoulder joint reflecting a longer-term movement to } \\
\text { prepared food and replacing an item which was sometimes difficult } \\
\text { to collect since joints are sometimes only available towards the end } \\
\text { of the week and on weekends. }\end{array}$ \\
\hline
\end{tabular}
01.1.6 Fruit
2128 Processed Fruit
Dried fruit

02.1.2 Wine

03.1.2 Garments

03.1.2 Garments

03.1.2 Garments

03.2.0 Footwear incl Repairs

04.3.1 Materials for Maintenance and Repair

06.1.2/3 Other Medical and5201 Personal Articles Therapeutic Equipment

08.2.0 Telephone and Telefax Equipment and Services

08.2.0 Telephone and Telefax Equipment and Services

09.1.1 Equipment for the Reception and Reproduction of Sound and Pictures

09.1.1 Equipment for the Reception and

Reproduction of Sound and Pictures

\subsubsection{Equipment for the} Reception and Reproduction of Sound and Pictures

09.3.1 Games, Toys and Hobbies

\subsubsection{Books}

09.5.2 Newspapers and Periodicals

12.1.2/3 Appliances and 5202 Chemists Goods Products for Personal Care

5105 Footwear

4106 DIY Materials

Equipment

6301 Audio-visual

Equipment

6301 Audio-visual

Equipment and Sports Goods

6304 Books and Newspapers

6304 Books and Newspapers

$\begin{array}{ll}\begin{array}{l}3104 \text { Wines \& Spirits Off } \\ \text { Sales }\end{array} & \text { Sparkling wine } \\ 5101 \text { Men's Outerwear } & \begin{array}{l}\text { Men's casual shirt, long/short } \\ \text { sleeve }\end{array}\end{array}$

5102 Women's Outerwear Women's blouse/shirt

5103 Children's Outerwear Girl's jacket

4404 Telephone Charges

4303 Electrical Appliances

Smart phone handsets

Flat panel TV 14"-22"

6303 Toys, Photographic

Women's high heeled shoes

Medium density fibreboard (MDF)

Spectacle frames with single vision lens

Mobile phone applications

Flat panel TV 23"-32"

Flat panel TV 33"+

Provincial newspaper

Dating agency fees
New item. Introduced to improve coverage of fruit products which have been identified as an under-represented area of the basket. It also reflects a distinct market and widens the coverage of processed fruit in the basket.

New item. Introduced to reflect spending on this type of item.

Replaces men's casual long-sleeved shirt with the aim of improving coverage during the summer months when the item was difficult to collect.

New item. A second blouse/shirt has been added to the basket reflecting the expenditure. This second item is aimed at the casual end of the market.

Replaces girls' winter \& girls' summer jacket. Introduced to improve coverage of the item across the year and to remove seasonal items whose prices have to be imputed during months when they are not available.

Replaces women's party shoes and women's high heeled sensible shoes due to footwear being over-represented in the basket.

Replaces hardboard as more representative of this area of the market and reflecting the relative spending on the two items.

Replaces spectacle frames without lens reflecting the way in which outlets increasingly do not price frames separately from lens.

Replaces mobile phone downloads. Introduced to capture price changes in this new expanding technology.

New item. Introduced to capture price changes in this new expanding technology.

New item. Introduced to capture the differing price movements from the wider range of screen sizes available, in particular the top end where they form part of home entertainment systems.

New item. Introduced to capture the differing price movements from the wider range of screen sizes available, in particular the top end where they form part of home entertainment systems.

New item. Introduced to capture the differing price movements from the wider range of screen sizes available, in particular the top end where they form part of home entertainment systems.

New item. Introduced to improve coverage of this underrepresented area of the basket. It also represents a distinct sector not previously covered within the class.

New item. Introduced to improve coverage of books which has been identified as an under-represented area of the basket.

Replaces morning \& evening provincial newspapers where the distinction has blurred over time.

New item. Introduced to improve coverage of this underrepresented area of the basket and reflects spending on hair conditioners.

New item. Prices of services in this residual category vary greatly and dating agency fees have been introduced to improve coverage of this under-represented class. 


\section{Table 4 Items removed from the basket in 2011}

(Note: 'low weighted' denotes an item with a CPI weight of less than 0.5 parts per thousand in 2010)

\begin{tabular}{lll}
\hline CPI Class & RPI Section & Dropped Item \\
\hline 01.1 .2 Meat & 2107 Pork & Pork shoulde
\end{tabular}

02.1.3 Beer

3102 Beer Off Sales

3201 Cigarettes

5101 Men's Outerwear

5102 Women's Outerwear

5102 Women's Outerwear

03.1.2 Garments

03.1.2 Garments

03.1.2 Garments

03.2.0 Footwear including 5105 Footwear Repairs

03.2.0 Footwear including 5105 Footwear Repairs

04.3.1 Materials for 4106 DIY Materials

Maintenance and Repair

06.1.2/3 Other Medical and5201 Personal Articles Therapeutic Equipment

08.2.0 Telephone and Telefax Equipment and Services

09.1.1 Equipment for the Reception and Reproduction of Sound and Pictures

09.1.1 Equipment for the Reception and

Reproduction of Sound

and Pictures

\subsubsection{Gardens, Plants} and Flowers

09.3.4/5 Pets, Related Products and Services

4306 Pet Care

09.5.2 Newspapers and Periodicals

09.5.2 Newspapers and 6304 Books and Periodicals
6304 Books and Newspapers

4404 Telephone Charges Mobile phone downloads

6301 Audio-visual Equipment

6301 Audio-visual Equipment

6305 Gardening Products

Rosebush

Vet fees for spaying a kitten

Morning provincial newspaper

Evening provincial newspaper

Flat panel TV 14"-25"

Flat panel TV 26"-42" Newspapers

Notes

Replaced by oven-ready joint reflecting a longer-term movement to prepared food. Also aimed at improving coverage since the pork shoulder was often only available towards the end of the week and on weekends.

Removed. The pack of four cans of lager has been removed to reduce the number of premium strength beers and rebalance the basket in this area.

Removed. Spending on vending machine cigarettes has been declining and current legislation will see these withdrawn during 2011.

Men's casual shirt, long sleeved Replaced by men's casual long or short sleeved shirt in order to improve coverage during the summer months.

Women's casual outer jacket (e.g Removed. Women's jackets remain represented in the basket by fleece) a separate women's casual outer jacket and a women's formal jacket.

Removed. This reflects the reduced weight of dresses in the basket. Dresses remain represented by a women's casual or formal dress.

Combined with girls' winter jacket to improve coverage of the item across the year and to remove a seasonal item whose price has to be imputed during months when it is not available.

Combined with girls' summer jacket to improve coverage of the item across the year and to remove a seasonal item whose price has to be imputed during months when it is not available.

Women's high heeled sensible Combined with women's high heeled party shoes since footwear shoes is over-represented in the basket.

Women's high heeled party shoe Combined with women's high heeled sensible shoes since footwear is over-represented in the basket.

Replaced by medium density fibreboard (MDF) reflecting the market share of the two items.

Replaced by spectacle frames with single vision lens since outlets increasingly do not price frames without lens.

Replaced by mobile phone applications.

Replaced by three sizebands of flat panel televisions to better measure differing price movements across the variety of television sizes available, particularly the top end where they form part of home entertainment systems.

Replaced by three sizebands of flat panel televisions to better measure differing price movements across the variety of television sizes available, particularly the top end where they form part of home entertainment systems.

Removed. A low weighted item in a section which is overrepresented.

Removed. This section of the basket is over-represented and in some cases cat spaying may be free. Vet fees remain represented by annual booster injections.

Combined with the evening provincial newspaper as the distinction has become blurred over time.

Combined with the morning provincial newspaper as the distinction has become blurred over time. 
- In other cases, the new items are direct replacements for similar products that leave the basket in 2011. For example, medium density fibreboard (MDF) replaces hardboard with the new product representing an increasing proportion of the market compared with the product it replaces. Another example here is a provincial newspapers item replacing separate morning and evening provincial newspapers with the main driver being the blurring of the distinction between the two separate items. Collection issues can also have an effect. For example, spectacle frames with single vision lens replace frames without lens as an increasing number of outlets do not price frames separate from the lens.

- It is important that the review of the basket considers not just the list of items to be priced, but also where the prices are collected. For example existing items are being collected from an increasing number of large supermarkets as they widen their product bases. These items include Blu-ray discs, computer games and reference books.

- The seasonality of some items in the basket is also reviewed. This year, separate items for girls' winter and summer jackets have been merged into one girls' jacket item. This should improve coverage across the year and remove two seasonal items for which prices have to be imputed during months when they are not available.

- Finally, as always, minor changes are made to the definitions in the item list caused by the availability of products. The main example this year is foreign exchange which is priced based on commission charges and changes in exchange rates. Previously the RPI item was based only on the cost of buying foreign currency but from 2011, it will be based on selling currency as in the CPI.

As noted earlier, it is important that growth in the overall size of the basket is limited each year so that production costs and processing times may be contained. A number of items therefore have been removed from the basket in 2011 to make room for the new additions. Some reflect reduced expenditure such as vending machine cigarettes which are set to be withdrawn during 2011 due to legislation. In other cases, the removal of items from the basket does not necessarily imply that the markets for these goods and services are very small or are declining significantly.

- Some items have been removed to make way for new additions to the basket within the same product grouping. For example, spectacles without lens have been removed in 2011 to be replaced by spectacles with lens, although both products represent spectacles. Similarly hardboard has been replaced by MDF. In other cases, items have been removed so that new items may be introduced covering distinct markets not previously represented explicitly within the product grouping. For example, the pork shoulder has been removed so that oven-ready joints can be represented.

- In some cases a product will still remain represented in the basket even if there is no longer an explicit item. For example, a women's premium branded dress has been removed but it is still 
covered by a more broadly defined casual and formal dress item. Similarly a women's casual outer jacket (for example fleece) is still covered by other casual and formal jacket items.

- Elsewhere, analysis suggested that there was scope to remove items from certain product groupings without any significant loss of precision in estimates of price changes overall. Within these groupings, those items with relatively low index weights or those items which are variants of other items have typically been chosen; examples include rose bushes and vet fees for spaying a kitten. In each case, it is judged that price changes for these goods remain adequately represented by those items that remain in the basket. The removal of items in such cases therefore represents a rebalancing of the basket, helping to offset the expansion of coverage in other product areas.

\section{Other changes}

One other change has already been introduced this year, with the January index published in February. This relates to the method used to measure prices of seasonal items. These are products likely to be unavailable to price for certain months of the year.

Previously the price index calculated in the last month that the product was available was carried forward until it became available again. The new method involves imputing the price forward each month using the average price movement of 'in-season' products. The 'in-season' products used are ones in the same classification group as the 'out of season' product but they are available to price when the 'out of season' product is not. The new method is internationally recognised as an improvement over the previous approach. This change is described in greater detail in a consultation document and a response to that consultation, 'Measurement of Seasonal Items within the Consumer Prices Index and Retail Prices Index ${ }^{5}$.

\section{Notes}

1. CPI indices are chain-linked first each January, when weights for CPI classes and higher level aggregates are updated, and again in February when changes to the basket are introduced and hence weights for individual item indices are reviewed.

2. RPI weights are based primarily on household spending estimates derived from the Living Costs and Food Survey, and relate to expenditures by private households only, excluding the top 4 per cent of households by income and those pensioner households mainly dependent on state benefits. CPI weights are based on National Accounts estimates of household final consumption consistent with the wider CPI population coverage (that is, all private households, residents of institutional households and foreign visitors to the UK).

3. At the extreme, if price changes for all the possible items that could be selected in a particular group were identical each month, it would be necessary to select only one of the items for inclusion 
in the basket. Price changes for this one item would be perfectly representative of price changes for the group as a whole.

4. Under CPI regulations, items should be included in the CPI where estimated consumers' expenditure is 1 part per thousand or more of all expenditure covered by the CPI; based on household final consumption data underpinning calculation of the $2011 \mathrm{CPI}$ weights, this is equivalent to around $£ 730$ million.

5. www.ons.gov.uk/about/consultations/closed-consultations/measurement-of-seasonal-itemswithin-the-cpi-and-rpi/measurement-of-seasonal-items-within-the-cpi-and-rpi.html

\section{Contact}

Public enquiries: 08456013034

CPI/RPI enquiries: 01633456900

Email: cpi@ons.gov.uk

\section{References}

Roe D and Fenwick D (2004) 'The New Inflation Target: the Statistical Perspective', Economic Trends No 602. Available at www.statistics.gov.uk/articles/economic_trends/ET602Roe.pdf

ONS (2010a) 'Consumer Price Indices - a Brief Guide'. Available at www.statistics.gov.uk/downloads/theme_economy/CPI-briefguide.pdf

ONS (2010b) 'Consumer Price Indices - Technical Manual'. Available at www.statistics.gov.uk/downloads/theme_economy/CPI_Technical_manual-2010.pdf

ONS (2010c) 'Differences between the RPI and CPI Measures of Inflation'. Available at www.statistics.gov.uk/downloads/theme_economy/cpi-rpi-information-note.pdf 